\title{
ESTUDIO COMPARATIVO DE LAS CURVAS DE CRECIMIENTO NCHS/OMS: EVALUACIÓN DEL ESTADO NUTRICIONAL E IMPLICANCIAS EN UN CENTRO DE SALUD FAMILIAR
}

\author{
COMPARATIVE STUDY OF NCHS/WHO GROWTH \\ CURVES: NUTRITIONAL STATUS EVALUATION AND \\ IMPLICATIONS IN A PUBLIC FAMILY HEALTH CENTER
}

\author{
María Angélica González S. (1), José Luís Pino V. (2) \\ (1) Universidad del Bío-Bío, Facultad Ciencias de la Salud y de los alimentos, \\ Departamento de Nutrición y Salud Pública, Chillán, Chile. \\ (2) Escuela de Nutrición y Dietética, Universidad del Mar, Sede Centro Sur, Talca, Chile.
}

\begin{abstract}
Chile changed from the CDC/NCHS to the new WHO standards in 2007 to evaluate growth in children less than 5 years of age. Our proposal was to determine the impact of this change on nutritional indicators. We studied 982 children aged less than 5 years in a public family health center in Chillan Viejo. Length/height-for-age, weightfor-length/height, and weight-for-age indicators were compared for both growth curves with the WHO Anthro program. Results showed significant differences in means for all anthropometric indicators $(p<0.0001)$, increased proportion of undernourished, overweight, and obese children, as well as a significant decrease of those with normal nutritional status. Consultation hours increased $27.7 \%$ and $41.1 \%$ for nutritionists and doctors, respectively. We concluded that the WHO standard represents more adequately optimum child growth, increases the proportion of children with nutritional excess and deficit leading to enhanced nutritional attention by health professionals. Key words: NCHS-WHO standards, nutritional evaluation, growth, anthropometry.
\end{abstract}

Este trabajo fue recibido el 29 de Septiembre de 2009 y aceptado para ser publicado el 12 de Mayo de 2010.

\section{INTRODUCCIÓN}

El crecimiento de lactantes y preescolares depende de factores genéticos y ambientales, los cuales se expresan en su máximo potencial cuando las condiciones son óptimas. Para medir su crecimiento se utilizan estándares de referencia los que evalúan la normalidad de su crecimiento, el cual es un objetivo prioritario de cualquier estrategia sanitaria. Por ello, las evaluaciones del crecimiento son indicadores "centinela" de la situación de salud y desarrollo socioeconómico de las comunidades (1). Los estándares de referencia se usan como instrumento para la acción, es así como en el ámbito individual, su monitoreo sirve para la identificación oportuna de desviaciones del crecimiento; en el ámbito local sirven para el tamizaje e implementación de acciones; y en el ámbito poblacional, el uso de la antropometría se utiliza en la selección de poblaciones para la intervención (2).

La antropometría es el método más utilizado para evaluar el tamaño, las proporciones, y la composición del cuerpo humano, por ser fácil de aplicar, de bajo costo y no invasivo. Permite predecir la nutrición, el rendimiento, la salud y la supervivencia. Por estas razones, se utiliza en el control de la salud y nutrición, así como en la selección de individuos y poblaciones a ser intervenidas (3).

El patrón recomendado por la OMS hasta el año 2006 para evaluar a los niños menores de 5 años, era el patrón internacional de crecimiento del National Center for Health Statistics (NCHS/OMS) (4). La referencia NCHS/OMS en menores de 2 años se basa en datos longitudinales del Instituto de Investigaciones Fels de Ohio, EEUU estudiados entre 1929 y 1975. Los datos correspondían a lactantes de descendencia europea alimentados fundamentalmente con sucedáneos de la leche materna, 
que residían en una zona geográfica limitada, de niveles socioeconómicos relativamente altos. Las mediciones fueron tomadas cada tres meses y el método analítico disponible en aquella época era inapropiado para describir el patrón y la variabilidad del crecimiento normal $(5,6)$.

La Organización Mundial de la Salud (OMS) a través de un grupo de trabajo sobre crecimiento infantil, determinó que la referencia NCHS/OMS era inapropiada para evaluar el crecimiento de lactantes sanos, al menos hasta los 12 meses, era necesario un nuevo patrón de crecimiento. La nueva curva debía describir cómo debería crecer los niños en condiciones ideales, por ello realizó el Estudio Multicéntrico de las Referencias del Crecimiento (MGRS en inglés), lo que fue una investigación multi-país cuyo propósito condujo a la elaboración de nuevos estándares de crecimiento para lactantes y niños pequeños. Este estudio tiene la peculiaridad de que fue concebido específicamente para elaborar un patrón seleccionando a niños saludables que vivieran en condiciones favorables para que los niños alcancen plenamente su potencial genético de crecimiento. Además, las madres de los niños seleccionados para la construcción de los patrones realizaban prácticas fundamentales de promoción de la salud, a saber, alimentar a los hijos con leche materna y no fumar (7).

La nueva referencia hace de la lactancia materna la "norma" biológica y establece el amamantamiento del lactante como el modelo normativo. Con un nuevo enfoque el estudio describe el crecimiento de los niños conforme a las prácticas sanitarias recomendadas y los comportamientos relacionados con resultados saludables. Por lo tanto, las curvas nuevas podrían considerarse como referencias normativas, a diferencia de las referencias tradicionales descriptivas basadas en muestras de niños con una sola representación geográfica, al margen de los comportamientos relacionados con la alimentación o con otros componentes. El MGRS se llevó a cabo en seis países que representan las principales regiones del mundo, abarcó 8.500 niños, fue una combinación de un estudio longitudinal de niños desde recién nacidos hasta los 24 meses de edad, y un estudio transversal de niños desde los 18 hasta los 71 meses (8). Las curvas OMS pueden ser utilizadas para evaluar a todos los niños, independiente de su origen étnico, condición social, o modalidad de alimentación (9).

Desde el año 1994 hasta el año 2006 el Ministerio de Salud de Chile (MINSAL), tenía como norma el uso de la referencia NCHS/OMS, dado que se ajustaba más a la realidad epidemiológica nacional existente a esa fecha. A contar del 2007 cambió a los nuevos estándares OMS, para el menor de 5 años (10), motivado por el desafío de la OMS, la cual recomienda a que cada país se incorpore a esta cruzada en pro de la salud infantil, integrando los esfuerzos de los Ministerios de Salud, grupos académicos y sociedades científicas, para lograr que cada niño(a) pueda crecer y desarrollarse en la forma más saludable que permite el conocimiento científico actual (11).

De acuerdo a estos antecedentes nos propusimos comparar el estado nutricional de un grupo de niños chilenos evaluados con las referencias NCHS/OMS y los nuevos estándares OMS, y medir su impacto en el diagnóstico nutricional y las implicancias en la atención nutricional de profesionales, con el fin de aportar antecedentes a nivel local y nacional que permitan orientar las estrategias necesarias oportunamente.

\section{SUJETOS Y MÉTODO}

La investigación consistió en estudiar a todos los niños en control, asistentes al Centro de Salud Familiar (CESFAM) "Dr. Federico Puga Borne" de la Comuna de Chillán Viejo. La muestra estuvo constituida por todos los niños menores de 5 años, con sus controles al día (n $=982$ niños). Se excluyeron a los niños con síndrome de Down, a RN pretérmino y a los inasistentes a controles durante el año 2006.

Los datos de género, peso, longitud/talla, fecha de nacimiento, fecha de evaluación, tipo de alimentación fueron extraídos del tarjetón de control de Salud del Programa del Niño del CESFAM. La información se obtuvo del último control. Para evaluar el estado nutricional se utilizó el módulo nutritional survey del programa WHO Anthro 2005 (12) expresado en desviación estándar (DE). Los criterios de diagnóstico nutricional utilizados fueron: peso para la edad $(\mathrm{P} / \mathrm{E})$, peso para la longitud/talla $(\mathrm{P} / \mathrm{T})$ y longitud/talla para la edad (T/E), recomendados por el Ministerio de Salud hasta 2006 con la referencia NCHS/OMS (13), y a partir del año 2007 por el nuevo estándar OMS (14).

Se estudió el impacto del cambio a través del aumento de las horas de consulta nutricional para médico y nutricionista con la nueva curva, de acuerdo a la norma técnica del Programa Infantil del MINSAL $(15,16)$ propuesta para el año 2007, aplicada en el Centro de Salud Familiar Dr. Federico Puga Borne de Chillán Viejo. Esta considera una cobertura del $100 \%$ a los niños con riesgo a desnutrir, desnutridos y obesos, y un $90 \%$ para los niños con sobrepeso; la concentración de 3 niños por hora para lactantes de 28 días a 5 meses en riesgo a desnutrición, desnutridos y para todos los niños con sobrepeso y obesidad; la concentración de 6 niños por hora para los infantes de 6 meses a 5 años 11 meses, en riesgo a desnutrición y desnutridos, el instrumento son los profesionales nutricionista y médico. 
El análisis estadístico se hizo usando comparación de medias, a través de Test de Chi cuadrado, t-Student para muestras pareadas, usando el programa estadístico Stata/SE 8.0, con un nivel de confianza del 95\%.

\section{RESULTADOS}

Se estudiaron 982 niños del CESFAM Chillán Viejo entre los meses Septiembre a Noviembre 2006, de ellos 498 eran varones y 484 mujeres. Las características generales de la muestra se pueden observar en la tabla 1, en ella se destaca las diferencias significativas encontradas en el peso de los niños, donde los hombres mostraron mayor peso que las mujeres $(\mathrm{p}<0.05)$. Las edades de los niños en control fueron categorizadas, encontrándose la mayor concentración entre 12 a 23 meses, y 48 a 60 meses; se destaca que el $13.7 \%(n=135)$ de los niños estudiados fueron menores de 6 meses.

El estado nutricional de los niños se expresó en puntuación Z (desviaciones estándar) por la referencia NCHS/OMS y por el nuevo estándar OMS, (tabla 2).

\section{TABLA 1}

\section{Características generales de los niños del CESFAM Dr Federico Puga Borne, comuna} Chillán Viejo 2006 (n= 982) de acuerdo a género (hombres 498, mujeres 484)

\begin{tabular}{lcccc} 
Variable & $\begin{array}{c}\text { Total } \\
\text { Prom } \pm \text { DE }\end{array}$ & $\begin{array}{c}\text { Hombre } \\
\text { Prom } \pm \text { DE }\end{array}$ & $\begin{array}{c}\text { Mujer } \\
\text { Prom } \pm \text { DE }\end{array}$ & P - value \\
\hline Peso (kg) & $12.67 \pm 4.51$ & $12.99 \pm 4.47$ & $12.36 \pm 4.52$ & $0.03^{*}$ \\
Talla (cm) & $85.34 \pm 16.41$ & $86.21 \pm 16.21$ & $84.45 \pm 16.59$ & NS \\
Edad (meses) & $26.59 \pm 17.54$ & $26.92 \pm 17.44$ & $26.26 \pm 17.65$ & NS \\
$0-5$ (meses) & 135 & $2.23 \pm 1.48$ & $2.98 \pm 1.76$ & NS \\
6 - 11 (meses) & 139 & $9.10 \pm 1.86$ & $8.95 \pm 1.79$ & NS \\
$12-$ 23 (meses) & 198 & $18.01 \pm 3.53$ & $17.85 \pm 3.55$ & NS \\
$24-$ - 35 (meses) & 175 & $29.18 \pm 3.18$ & $29.16 \pm 3.58$ & NS \\
$36-47$ (meses) & 154 & $40.87 \pm 3.44$ & $40.53 \pm 3.23$ & NS \\
$48-60$ (meses) & 181 & $52.98 \pm 3.66$ & $52.89 \pm 3.69$ & NS \\
* T-Student Test paired & & & &
\end{tabular}

\section{TABLA 2}

Indicadores del estado nutricional de acuerdo a NCHS y OMS según género de los niños del CESFAM Dr Federico Puga Borne, comuna Chillán Viejo 2006

\begin{tabular}{|c|c|c|c|}
\hline Indicador & NCHS & OMS & P-value \\
\hline Peso/Edad & $0.34 \pm 1.11 *$ & $0.42 \pm 1.02$ & $<0.0001^{\mathrm{a}}$ \\
\hline Hombres & $0.30 \pm 1.12$ & $0.42 \pm 1.04$ & $<0.0001^{\mathrm{a}}$ \\
\hline Mujeres & $0.39 \pm 1.11$ & $0.42 \pm 0.99$ & $<0.001^{\mathrm{a}}$ \\
\hline Peso/Talla & $0.50 \pm 1.04$ & $0.68 \pm 1.07$ & $<0.0001^{\mathrm{b}}$ \\
\hline Hombres & $0.45 \pm 1.05$ & $0.55 \pm 1.03$ & $<0.0001^{\mathrm{b}}$ \\
\hline Mujeres & $0.71 \pm 1.11$ & $0.66 \pm 1.02$ & $<0.0001^{\text {b }}$ \\
\hline Longitud/Estatura/Edad & $0.01 \pm 1.07$ & $-0.12 \pm 1.13$ & $<0.0001^{\text {b }}$ \\
\hline Hombres & $-0.04 \pm 1.06$ & $-0.16 \pm 1.15$ & $<0.0001^{\mathrm{b}}$ \\
\hline Mujeres & $0.06 \pm 1.08$ & $-0.09 \pm 1.11$ & $<0.0001^{\mathrm{b}}$ \\
\hline
\end{tabular}


Al comparar los resultados se descubrieron diferencias significativas entre ambas referencias, donde las evaluaciones por curvas OMS fueron más exigente en todos los indicadores, contrastes que se hacen más evidentes en los hombres. Estas diferencias se destacan mejor en la figura 1, donde la curva de los niños controlados en el CESFAM de Chillán Viejo por el indicador P/T se desplaza hacia la derecha, en ambas referencias, siendo más acentuada en la curva OMS, lo que demuestra que ésta califica a más niños con estado nutricional en exceso que la curva NCHS/OMS. Al estudiar este indicador por edades de los niños se pudo apreciar que estas diferencias

\section{FIGURA 1}

Puntaje $Z$ de peso para longitud/talla de niñas de acuerdo a curvas NCHS y OMS.
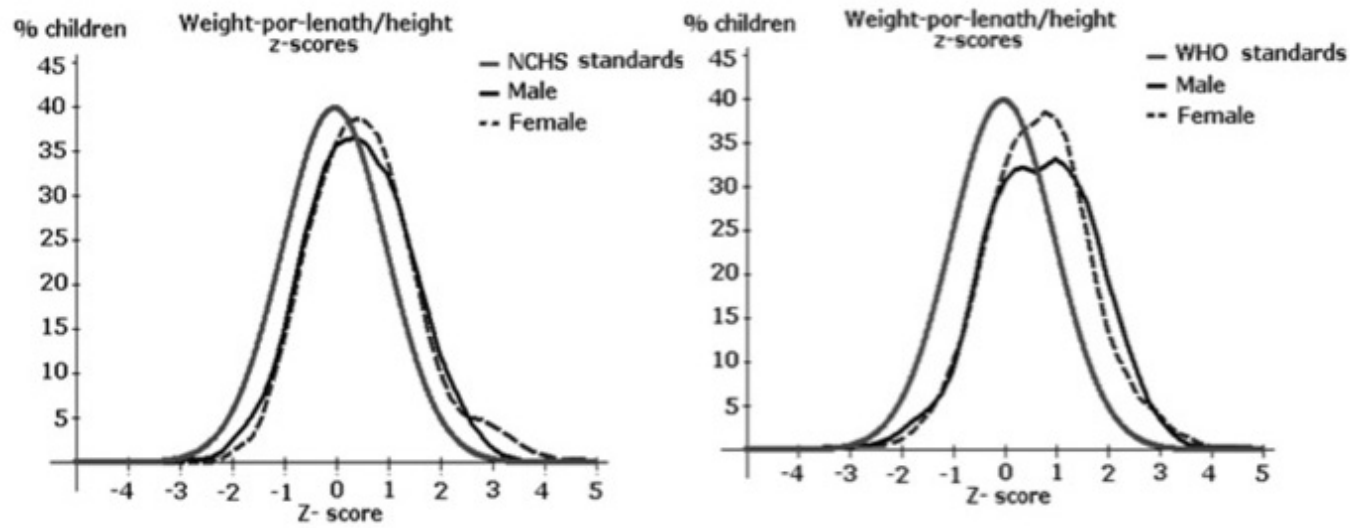

\section{FIGURA 2}

Puntaje $Z$ de Longitud/Estatura para la edad de niños del CESFAM Dr Federico Puga Borne, categorizado por edad de acuerdo a curvas NCHS y OMS, Chillán Viejo, 2006.

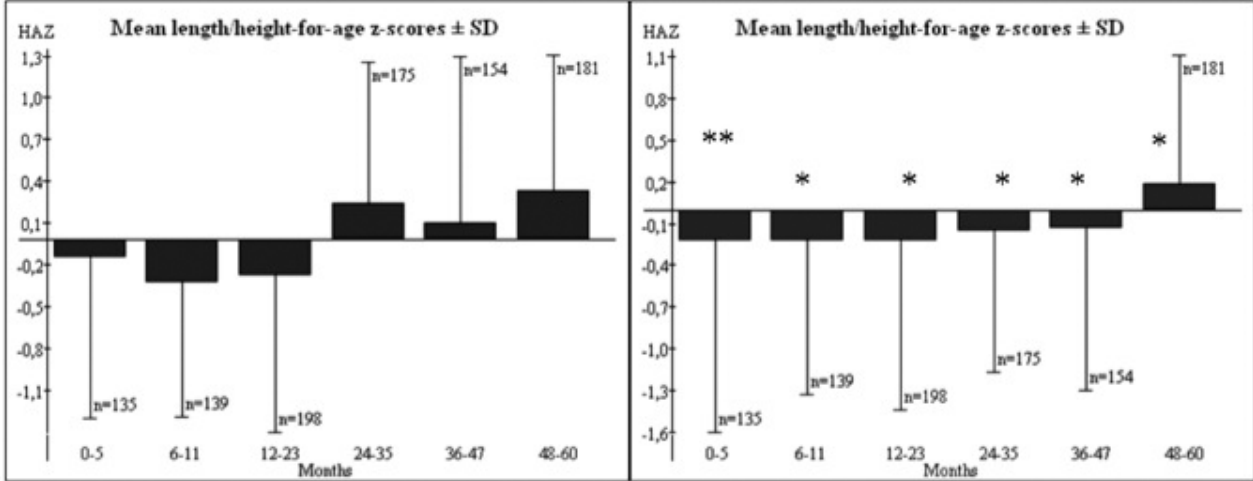

${ }^{* *} \mathrm{p}<0.0003 \quad * \mathrm{p}<0.0001$ 
de evaluación eran significativas $(\mathrm{p}<0.001)$ en todas las edades categorizadas.

De acuerdo al indicador $\mathrm{T} / \mathrm{E}$, con la referencia NCHS/OMS los niños aparecen normales, sin embargo con el nuevo estándar OMS, esta se desplaza hacia la izquierda, lo que nos indica que el éste es más exigente en talla. Como se puede observar en la figura 2, al categorizar por edad el indicador $\mathrm{T} / \mathrm{E}$, los niños clasificados por el estándar OMS, aparecen con valores negativos hasta los 48 meses de edad, sin embargo con la referencia NCHS/OMS solo hasta los 12-24 meses. Respecto a la talla baja $(<2 \mathrm{DE})$ aumenta de $2.2 \%$ a $3.97 \%$, el porcentaje de niños normales se mantiene en $93.28 \%$ (NCHS/=MS) vs. $92.06 \%(\mathrm{OMS})$, y los niños con talla alta disminuyen de un $4.48 \%$ a un $3.97 \%$.

De acuerdo al diagnóstico nutricional según norma MINSAL se puede apreciar que (tabla 3 ) con la nueva referencia OMS la estadística nutricional del CESFAM de Chillán Viejo, disminuye el Riesgo de desnutrir en 0,6 puntos porcentuales, y los niños normales en 13,5 puntos porcentuales; aumenta la desnutrición en 0.5 puntos porcentuales, el sobrepeso en 9,6 puntos porcentuales y la obesidad en 4 puntos porcentuales.

Los niños menores de 6 meses con lactancia materna

\begin{tabular}{|c|c|c|c|c|c|}
\hline \multicolumn{6}{|c|}{$\begin{array}{l}\text { Diagnóstico nutricional de niños del CESFAM Dr. Federico Puga Borne de acuerdo a parámetros } \\
\text { NCHS y OMS, comuna Chillán Viejo, } 2006(n=982)\end{array}$} \\
\hline \multirow[t]{2}{*}{ Diagnóstico nutricional } & \multicolumn{2}{|c|}{ NCHS } & \multicolumn{2}{|c|}{ OMS } & \multirow[t]{2}{*}{ Valor $\mathbf{p}$} \\
\hline & $\mathbf{n}$ & $\%$ & $\mathbf{n}$ & $\%$ & \\
\hline Desnutrición & 2 & 0,2 & 7 & 0,7 & N.S. \\
\hline Riesgo desnutrir & 50 & 5,1 & 44 & 4,5 & N.S. \\
\hline Normal & 663 & 67,5 & 530 & 54 & $<0.0001$ \\
\hline Sobrepeso & 202 & 20,6 & 297 & 30,2 & 0.0168 \\
\hline Obesidad & 65 & 6,6 & 104 & 10,6 & N.S. \\
\hline
\end{tabular}

\section{FIGURA 3}

Horas Requeridas para consultas de atención de médico y nutricionista por norma técnica según diagnóstico nutricional OMS y NCHS de niños menores de 5 años, CESFAM Chillán Viejo, 2006.

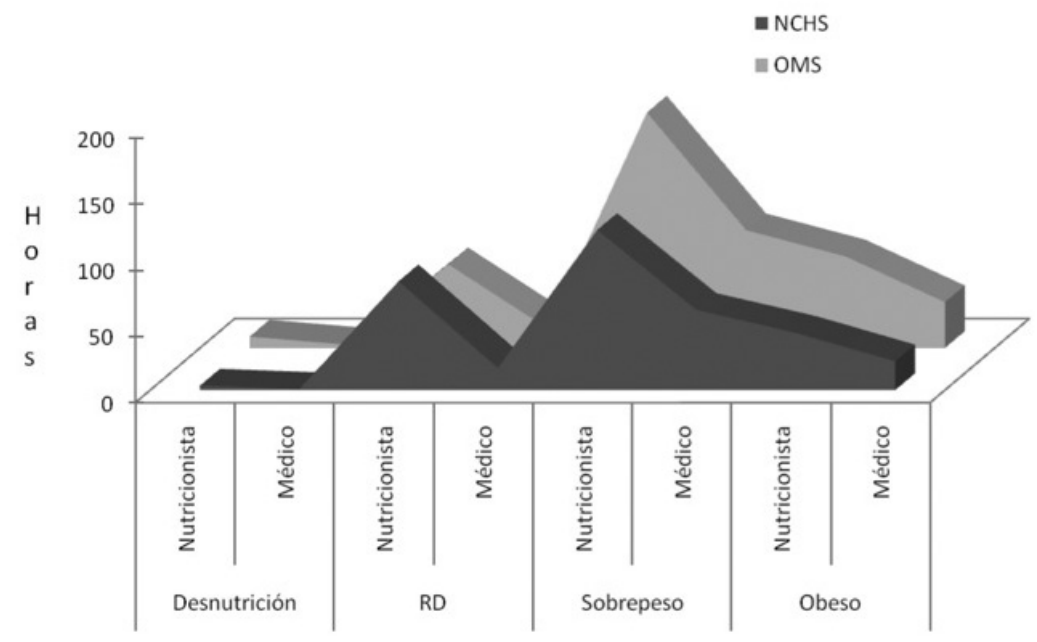


exclusiva eran catalogados como niños normales según los parámetros del OMS/NCHS y norma MINSAL 2006, ahora con los nuevos criterios OMS el diagnóstico real del estado nutricional de los niños cambió a $52.17 \%$ normal, $2.17 \%$ desnutrición, $9.78 \%$ riesgo de desnutrición, $28.26 \%$ sobrepeso y $7.61 \%$ obesidad.

Se hizo una comparación de las horas de consulta de atención de los profesionales nutricionista y médico (figura 3) requeridas, según las normas técnicas de atención primaria, entre las demandadas por la referencia NCHS/OMS con las del estándar OMS, encontrándose que la consulta de nutricionista aumentó en un $27.7 \%$ y la del médico en $41.1 \%$, dada principalmente para la atención de niños con sobrepeso y obesidad. Para ambos profesionales disminuyen las horas de consulta para el niño con riesgo de desnutrición y aumenta para los niños desnutridos, para sobrepeso y obesidad.

\section{DISCUSIÓN}

Se comparó el estado nutricional de los niños menores de 5 años del Centro de Salud Familiar "Dr. Federico Puga Borne" de la Comuna de Chillán Viejo, por las referencias NCHS/OMS usadas hasta el año 2006 con los nuevos estándares OMS recomendadas por el MINSAL a partir del 2007; en él se estudiaron 982 niños menores de 5 años. De acuerdo a las nuevas curvas OMS las diferencias de medias en la puntuación $\mathrm{Z}$ de PE, PT y TE, son semejantes a los obtenidas por el estudio multicéntrico realizado por la Organización Mundial de la Salud, la cual reconoce que existen importantes diferencias con el patrón del NCHS/OMS que varían según la edad, el sexo, la medida antropométrica y la curva específica de percentiles o de puntuaciones z, además reflejan diferencias, no sólo en las poblaciones que se han utilizado, sino también en las metodologías aplicadas para construir los dos conjuntos de curvas de crecimiento (6).

La media del puntaje $\mathrm{Z}$ de PE fue superior en la curva OMS que en la NCHS/OMS, igual a lo encontrado en el estudio Muticéntrico de la OMS, donde las medias de los estándares de las curvas OMS son mayores para los niños pequeños y disminuyen después de los 8 meses de edad, además las desviaciones estándares superiores son marcadamente más elevadas que las desviaciones estándares bajas. A partir de los 4 meses la velocidad de ganancia de peso es menor en las curvas OMS que en las de la NCHS/OMS $(6,17)$, por ello las curvas OMS capturan la ganancia de peso y el crecimiento rápido de la primera infancia, incluyendo la pérdida fisiológica de peso de los primeros días de vida 6. Diferencias que se deberían al tipo de alimentación de los niños de de la referencia NCHS/OMS los cuales fueron alimentados con fórmula, en cambio los niños de los estándares OMS fueron todos alimentados con leche materna (18).

Respecto a la media del puntaje $\mathrm{Z}$ de TE, las diferencias encontradas entre el estándar OMS y la referencia NCHS/OMS fue marcadamente significativa, demostrándose ser más exigente en longitud/talla con la nueva curva OMS, en especial para los niños menores de 48 meses, en donde los valores tanto para hombres como mujeres estuvieron por debajo de -1DE. Los promedios de los niños del Centro de Salud fueron negativos hasta los 24 meses por las curvas NCHS/OMS y hasta los 47 meses por las curvas OMS. Lo anterior podría deberse a que en el estándar OMS el patrón para el crecimiento lineal tiene una parte basada en la longitud (longitud para la edad, de 0 a 24 meses) y otra basada en la estatura (estatura para la edad, de 2 a 5 años). Las dos partes se construyeron utilizando el mismo modelo, pero las curvas finales reflejan la diferencia media entre la estatura en posición recostada y la estatura en posición vertical. De Onis demostró que los estándares de la OMS son más estrechos, y la duración de las medianas son similares a las de la NCHS/OMS 2000, salvo en las edades de 24 a 36 meses que son mayores en las nuevas curvas. El estándar de la OMS, demostró que los niños son, en promedio, más altos que los de la tabla de NCHS/ OMS lo cual debería disipar la preocupación de que los niños amamantados podrían no llegar a cumplir con su potencial de para el crecimiento de tejido libre de grasa, debido a la ingesta marginal de la energía, la proteínas y / o otros nutrientes (16).

Se encontraron diferencias entre ambas curvas en la comparación de la media del puntaje z de PT, esta podría deberse a el hecho de que el indicador combine diferentes velocidades para las dos mediciones (peso y longitud/estatura) en edades que se solapan, lo cual explica probablemente los ligeros altibajos en los patrones definitivos de la OMS, que se observan también en otras referencias (6). Entre los niños más bajos, la DE inferior están encima de los valores NCHS/OMS. Para los niños más altos, las nuevas curvas están por debajo de los valores NCHS/OMS. Los estándares de la OMS abarcan una gama más amplia de mediciones de talla que la NCHS/OMS, y muestra que los niños por la NCHS/ OMS son generalmente más pesados de los incluidos en la muestra de la OMS, ello explica que la prevalencia de obesidad es mayor en las curvas OMS.

Con los nuevos estándares de la OMS los niños del CESFAM en riesgo de desnutrir disminuyeron de un $5.1 \%$ (NCHS/OMS) a un 4,5\%, sin embargo aumentó la cifra de niños desnutridos de un $0.2 \%$ a un $0.7 \%$, confirmando lo señalado por De Onis, con las nuevas curvas OMS aumenta la prevalencia de niños con desnu- 
trición y los con talla baja $(6,19)$. La población infantil estudiada del CESFAM de la comuna de Chillán Viejo muestra diferencias con las estudiadas por Onyango y cols. (20) quienes compararon poblaciones de 4 países, el porcentaje de niños con talla baja de nuestro estudio fue menor $3.97 \%$; el que más se asemejaba es Italia con un $6.9 \%$, sin embargo, los niños de talla alta son superiores en la población de Italia (37.3\%) vs 3.97. La mediana de la TE de los niños del CESFAM comparado con la el estándar OMS mostró que un $92.06 \%$ de los niños se encontraba clasificado como normal, lo que demuestra que los esfuerzos hechos en los últimos años por el MINSAL por mejorar la nutrición de los lactantes como fortificar la leche está dando sus frutos.

Los resultados presentados evidencian un aumento importante de la malnutrición por exceso (sobrepeso y obesidad) en los niños menores a 5 años, por ello es de vital importancia tomar las acciones necesarias para afrontar esta situación. La labor educativa del profesional nutricionista es fundamental para la difusión de una alimentación balanceada y adecuada a los requerimientos de los niños $(21,22)$. Los talleres que por norma ministerial se deben realizar, serán una herramienta esencial para combatir la malnutrición por exceso mediante persuasión a las madres para cambiar los estilos de vida y forma de alimentación (12). Además, la velocidad de crecimiento de los estándares OMS son muy útiles, para la evaluación temprana del riesgo de sobrepeso en los lactantes, por ello, son un aporte a la gestión temprana de este problema de salud pública prevalente y preocupante que va en aumento. En lugar de esperar para detectar los riesgos cuando el problema ya se haya presentado, las referencias de velocidad facilitan la detección de niños en riesgo de estar desnutridos o sobre nutridos (23).

También se ha expresado que las curvas NCHS/ OMS son inadecuados para los niños amamantados sanos (24). Cabe destacar que con la nueva referencia OMS, el diagnostico de los niños menores de 6 meses con lactancia materna exclusiva será efectivo y no se diagnosticaran como niños normales (13). Según los resultados del CESFAN solo el 52\% de los lactantes se encontraban con un estado nutricional normal, el resto de ellos necesitaba atención nutricional, tanto para el déficit como para el exceso de peso.

El sistema de evaluación y vigilancia nutricional implementado por el Ministerio de Salud desde el año 1994, permite disponer de información sistemática para todos los indicadores referidos y contar con datos de nuestra población actualizados permanentemente, lo que a su vez ha permitido programar las intervenciones nutricionales necesarias (25). La Consulta Nutricional, está destinada para los niños que tienen su estado nu- tricional alterado ya sea por déficit como por exceso y esta la realiza los profesionales médico y nutricionista (14), nuestro estudio demostró que la demanda de horas para ambos profesionales aumentó.

Se concluye que con las nuevas curvas OMS la proporción de niños desnutridos aumenta, lo mismo pasa con el sobrepeso, obesidad y talla baja, y disminuye el porcentaje de niños en riesgo de desnutrir; como consecuencia, aumenta la demanda de horas nutricionista y médico. Sin embargo, de acuerdo al estudio multicéntrico la evaluación del crecimiento de los niños es más real, ya que se expresa todo el potencial genético al ser comparado con niños alimentados al seno materno y en condiciones de salud y ambientales ideales.

\section{RESUMEN}

El año 2007 Chile cambió la referencia NCHS por los nuevos estándares OMS, para evaluar el crecimiento de los menores de 5 años. Por ello, nos propusimos determinar el impacto del cambio en los indicadores nutricionales. Se estudiaron 982 menores de 5 años del Centro de Salud Familiar de Chillan Viejo, por los indicadores Talla/Edad, Peso/Talla y Peso/Edad, comparados por ambas curvas, en el programa WHO Anthro. Los resultados mostraron diferencias significativas en las medias de todos los indicadores antropométricos $(\mathrm{p}<0.0001)$, aumentó la proporción de niños desnutridos, sobrepeso y obesos, y disminuyeron significativamente los niños con estado nutricional normal. Las horas de consulta nutricional para nutricionistas y médicos aumentaron un $27.7 \%$ y $41.1 \%$ respectivamente. Concluimos que el estándar OMS representa mejor el crecimiento infantil óptimo, aumenta la proporción de niños con exceso y con déficit nutricional, lo que lleva a mayor atención nutricional de los profesionales.

Palabras clave: curvas NCHS-OMS; evaluación nutricional; crecimiento; antropometría.

Dirigir la correspondencia a:

Profesora

María Angélica González Stäger

Facultad Ciencias de la Salud y de los Alimentos

Departamento de Nutrición y Salud Pública

Universidad del Bío-Bío

Chillán - Chile

Avda. Andrés Bello sin Número

Chillán

Fono: $42-253121$

Fax: $42-253142$

E-mail: magonzal@ubiobio.cl

Agradecimientos: Agradecemos al personal que 
trabaja en el CESFAM Chillán Viejo las facilidades otorgadas para llevar a cabo esta investigación.

\section{BIBLIOGRAFÍA}

1. Atalah E. Una nueva referencia internacional de crecimiento infantil. ATALAH S, Eduardo. Una nueva referencia internacional de crecimiento infantil. Rev. chil. pediatr., Santiago, v.78, n.2, abr. 2007. Disponible en <http://www.scielo.cl/scielo.php?script=sci_arttext\&pid=S037041062007000200011\&lng=es\&nrm=iso $>$. accedido en09 sept. 2009. doi: 10.4067/S0370-410620070 00200011.

2. Rivera J. Referencia del crecimiento: un instrumento en el_contexto_de_la_atención primaria de salde http./www paho_grg/spanish/ad/fch/nu/ MEX04_Reunion.htm 2004 Consultado 9/09/2009.

3. De Onis M, Habicht J. Anthropometric reference data for international use: recommendations from a World Health Organization Expert Committee. Am J Clin Nutr 1996;64:650-8.

4. Physical status: the use and interpretation of anthropometry. Report of a WHO Expert Committee. World Health Organ Tech Rep Ser 1995; 854:161262.

5. Hamill PV, Drizd TA, Johnson CL, et al. NCHS growth curves for children birth-18 years United States.Vital Health . 1977; Stat 11(165).

6. Hamill PV, DrizdTA, Johnson CL, et al. NCHS growth charts. MonthlyVital Statistics Report 1976; 25 (3 suppl).

7. WHO Multicentre Growth Reference Study Group. WHO Child Growth Standards: Length/height-forage, weight-for-age, weight-for-length, weight-forheight and body mass index-for-age: Methods and development. Geneva: World Health Organization, 2006 (312 pages).

8. De Onis M, Garza C, Victora C, Onyango A, Frongillo E and Martines J. The WHO Multicentre growth reference study: Planning, study design, and methodology. Food Nutr Bull 2004; 25:1 (Suppl 1): S15- S26.

9. De Onis, M, Onyango AW, den Broeck J, Cameron Chumlea W Martorelli R. Measurement and standarization protocols for anthropometry used in the construction of a new international growth reference. Food and Nutrition Bulletin. 2004; 25:1 (Suppl 1): S27-S36.

10. MINSAL_Sección alimentos y Nutrición http. f www.minsal.cl/ici/nutricion/nutricion.html. consultado el 15/06/2007.

11. De Onis M, Habicht JP. Anthropometric reference data for international use: recommendations from a WHO Expert Committee. Am J Clin Nutr 1996; 64: $650-8$.

12. WHO Anthro 2005 software and macros http. www.who.int/childgrowth/software/en/index.html. Consultado 20/08/2007.

13. MINSAL. Norma para el manejo ambulatorio de la malnutrición por déficit y exceso en el niño(a) menor de 6 años. Depto. Ciclo Vital y Nutrición División Prevención y Control de Enfermedades. 2005.

14. MINSAL. Modificación norma manejo ambulatorio de la malnutrición por déficit y exceso en el niño(a) menor de 6 años según nuevos estándares antropométricos OMS 2006. Departamento alimentos y nutrición División Políticas Públicas Saludables y Promoción. Ministerio de Salud. Enero 2007.

15. MINSAL. Norma para el Manejo ambulatorio De la malnutrición Por déficit y exceso En el niño(a) menor de 6 años. 2007.

16. MINSAL. Normas técnicas 2007. Ciclo vital 0-9 años. Equipo ciclo vital 0-9 años Servicio de Salud Nuble.

17. De Onis M, Garza C, Onyango A, Borghi E. Comparison of the WHO Child Growth Standards and the CDC 2000 Growth Charts. Symposium: A New 21st-Century International Growth Standard for Infants and Young Children. J Nutr 2007. 137;144-8.

18. WHO Multicentre Growth Reference Study Group. WHO Child Growth Standards based on length/ height, weight and age. Acta Paediatr (Suppl) 2006; 450:76-85.

19. De Onis M, Onyango E, Borghi E, Garza C, Hong Yang H, for the WHO Multicentre Growth Reference Study Group. Comparison of the World Health Organization (WHO) Child Growth Standards and the National Center for Health Statistics/WHO international growth reference: implications for child health programmes. Public Health Nutrition 2006 ; 9(7), 942-947.

20. Onyango A, De Onis M, Caroli M, Shah U, Sguassero Y, Redondo N, Carroli B. Field-Testing the WHO Child Growth Standards in Four Countries. Symposium: A New 21st-Century International Growth Standard for Infants and Young Children. J Nutr 2007:149-152.

21. Nutrición XXI, Instituto de Nutrición y Tecnología de los Alimentos (INTA), No 10, junio-julio 2003, P 13.

22. L. Edmunds, E. Waters, E. Elliott, Manejo de la obesidad infantil basado en la evidencia, BRITISH MEDICAL JOURNAL, Departamento de Salud 
Pública de Universidad de Oxford, Nº 997 p 33.

23. Garza C, De Onís M, por el Grupo del Estudio Multicentro sobre las Referencias del Crecimiento de la OMS. Justificación para la elaboración de una nueva referencia internacional del crecimiento. Food Nutr Bull 2004, 25 (1) S5-S14.
24. WHO. The World Health Organization's infantfeeding recommendation. Wkly Epidemiol Rec 1995:70:119-20.

25. MINSAL. Norma para el manejo ambulatorio de la malnutrición por déficit y exceso en el niño(a) menor de 6 años. 2004. 